\title{
Optimizing the Electrical Energy Conversion Cycle of Dielectric Elastomer Generators
}

\section{Citation}

Shian, Samuel, Jiangshui Huang, Shijie Zhu, and David R. Clarke. 2014. "Optimizing the Electrical Energy Conversion Cycle of Dielectric Elastomer Generators." Advanced Materials 26 (38) (August 11): 6617-6621. doi:10.1002/adma.201402291.

\section{Published Version}

doi:10.1002/adma.201402291

\section{Permanent link}

http://nrs.harvard.edu/urn-3:HUL.InstRepos:12559504

\section{Terms of Use}

This article was downloaded from Harvard University's DASH repository, and is made available under the terms and conditions applicable to Open Access Policy Articles, as set forth at http:// nrs.harvard.edu/urn-3:HUL.InstRepos:dash.current.terms-of-use\#OAP

\section{Share Your Story}

The Harvard community has made this article openly available.

Please share how this access benefits you. Submit a story.

\section{Accessibility}




\section{Optimizing the Electrical Energy Conversion Cycle of Dielectric Elastomer Generators}

Samuel Shian*, Jiangshui Huang, Shijie Zhu, and David R. Clarke

S. Shian, J. Huang, S. Zhu, D.R. Clarke

School of Engineering and Applied Sciences, Harvard University, Cambridge, MA 02138, USA

E-mail: sshian@seas.harvard.edu

\section{J. Huang}

Present address: Schlumberger Research Center, Sugarland, TX 77478, USA

S. Zhu

Fukuoka Institute of Technology, Japan.

Keywords: energy harvesting, soft matters, dielectric elastomers, electromechanical conversion cycle, generators

Most common dielectric elastomer (DE) generators are, in effect, voltage upconverters, using mechanical energy to increase the electrical energy of charge on a soft capacitor. They operate on an electromechanical cycle in which an elastomer sheet is first stretched, the surfaces charged and then the elastomer is allowed to return to its initial thickness under open circuit conditions, increasing the voltage between the charges which are then conducted away before the cycle is repeated. The intrinsic advantages of high energy density, lightweight, direct linear motion, flexibility in speed, and possible good elastic impedance matching ${ }^{[1-3]}$ all make these dielectric elastomer generators attractive. However, the timing of the charge placement onto the elastomer and extraction play important roles in the generator performance. Two of the important figures of merit for comparing these generators are their energy density in one electromechanical cycle over the mass of active elastomer and energy conversion efficiency. For these reasons, several harvesting schemes have been proposed to maximize the energy density or the electrical energy gain ${ }^{[2,4-7]}$ but the optimum one has yet to be identified.

In one of the earliest publications, Pelrine ${ }^{[2]}$ proposed the use of constant charge which is relatively easy to implement. Additionally, he analyzed a combination of breakdown limited condition and "field-supported" or loss of tension prevention that were considered to 
produce higher energy density than the constant charge scheme. However, it was not clear how these high energy density schemes he described could be implemented in practice. Subsequently, Koh, Zhao and Suo ${ }^{[4]}$ provided a rigorous analysis of the energy generation cycle using dielectric elastomers by imposing boundary conditions dictated by failure modes of the materials, i.e., electrical breakdown (EB), electromechanical instability (EMI), loss of tension (LT), and rupture of the elastomer by excessive stretching. Their work provides useful guidance by describing these boundary conditions in both the work-conjugate planes of force versus stretch and of the voltage versus charge. The latter is redrawn in Figure 1(a) from Koh et al. ${ }^{[8]}$ for acrylic dielectric elastomers under equibiaxial loading. Note that Gent hyperelastic model $^{[9]}$ is used in this work with shear modulus, $\mu$, and stretch limit, $J_{\text {lim }}$, of $66 \mathrm{kPa}$ and 96, respectively. They also proposed an energy harvesting scheme using constant voltages for both input and output and shown in Figure 1(a) by the rectangle $d-e-f-g-d$. Obviously, the output voltage is higher than the input voltage for a positive net electrical generation. Because of its simplicity, this harvesting scheme has been used by several authors in various DE generators. ${ }^{[10-15]}$ However, the maximum energy density harvested using the constant voltage schemes is significantly lower, less than one-half, than the maximum possible energy density based on analysis of the individual failure modes. ${ }^{[4,12]}$

This letter describes a harvesting scheme that optimizes the energy density of dielectric elastomer generators by controlling the rate of charge transport in an electromechanical cycle. It is shown that when represented in the voltage-charge workconjugate plane, the scheme is capable of a high harvesting energy density, approaching the theoretically achievable for a given material. The effectiveness of the scheme is verified experimentally and produces the highest energy density yet reported for DE generators made of acrylic materials. The scheme is implemented with equibiaxial loading, which has been shown to maximize the capacitance change and consequently the energy density in comparison with other mode of deformations, i.e., pure shear and uniaxial. ${ }^{[12]}$ A subtle but 
important point is that equibiaxial tension is equivalent to a perpendicular uniaxial compression since elastomers are incompressible. In this configuration, the compression strain produced by the Maxwell stress from the charges on the opposite surfaces is directly transferred into the lateral strain, and vice versa, with minimal mechanical constraint.

The proposed harvesting cycle seeks to maximize in a simple cycle the enclosed area in the voltage-charge plane by operating the DE close to the loci of the limits imposed by the possible failure mechanisms. It is shown as the triangle $A-B-C$ in Figure 1(a). A simplified electrical circuit showing the essential components for performing the triangular cycle is shown in Figure 1(b) and the harvesting cycle is described in the following with reference to both Figure 1(a) and 1(b).

The cycle starts at state A where the DE has no charges on its electrodes. The DE is then stretched to a maximum value somewhat lower than its rupture stretch value. The latter is typically calculated from uniaxial tensile test using a constitutive equation such as Gent hyperelastic material model. ${ }^{[9]}$ Due to technical difficulties, such as edge effects, we used a workable maximum stretch $\left(\lambda_{\max }\right)$ of 5.5 instead the calculated rupture stretch $\left(\lambda_{\text {rupture }}\right)$ of $7 . \mathrm{A}$ diode D1 is used to prevent charge flowing from the transfer capacitor $(C p)$ onto the DE during the stretching process and whenever $\Phi_{D E}<\Phi_{C p}$. Once the DE has been stretched to a pre-set value, it is connected to a constant current power supply by activating switch S1. During the charging step the DE changes from state A until the potential across the dielectric equals that of the supply and state B is reached. At this stage, a preset input voltage, $\Phi_{i n}$, from the power supply is attained, and the charge and the voltage are related by the capacitance of the DE given by the state equation $Q_{D E}=C_{D E} \Phi_{i n}$, where $C_{D E}$ remains nearly constant at $C_{D E}=C_{o} \lambda_{\max }^{4},\left(C_{o}\right.$ is the initial capacitance of the unstretched DE). Then, in the next step, the mechanical loading is reduced and the elastic energy stored in the elastomer decreases until state $\mathrm{C}$ corresponding to the minimum stretch. The minimum stretch 
determines the minimum capacitance and, based on our previous work ${ }^{[12]}$, the minimum practical value is $\lambda_{\min }=2$. Although a value as small as $\lambda_{\min }=1$ is possible, the inherent viscoelasticity of the elastomer would require an appreciable extra time to reach this small value, decreasing the attainable power. During this mechanical unloading, the thickness of the elastomer increases, and the elastic strain energy is converted into electrical energy by increasing the potential across the elastomer. This, in turn, drives charge to the transfer capacitor. Since the capacitor has finite capacitance, the additional charge increases the voltage across it. As the DE further relaxes, more charges on the DE transfer to $C p$ and at the same time the potential of both DE and $C p$ increase. The increase in voltage is linear with the amount of charge transferred. Assuming charge conservation, the slope of line B-C is inversely proportional to the capacitance of the transfer capacitor or $\Delta \Phi / \Delta Q=-1 / C p$. When the capacitance of $C p$ is zero or no transfer capacitor present, the voltage increases vertically, and when capacitance of $C p$ is infinite, the voltage remains constant. To maximize the energy conversion, the value of the fixed capacitor $C p$ is chosen so that line $\mathrm{B}-\mathrm{C}$ almost touches the failure limit lines, which are typically either those corresponding to the electrical breakdown voltage (EB) or the electromechanical instability (EMI) curves in Figure 1 (a).

At state $\mathrm{C}$, the $\mathrm{DE}$ is at the highest voltage, which is coincidentally in its minimum stretch condition, $\lambda_{\min }$, and most of its charge has been transferred to the capacitor. The remaining charge is a function of minimum capacitance at this smallest stretch. For equibiaxial loading, the minimum capacitance of the DE is less than $2 \%$ of its maximum capacitance at the stretch equal to $5.5,{ }^{[12]}$ which means that the line $\mathrm{C}-\mathrm{A}$ is almost coincident with the voltage axis. Nevertheless, when switch $\mathrm{S} 2$ is activated, the charge on the storage capacitor $C p$ and the remaining charge on the DE can be harvested by connecting to a storage device. The total electrical energy generated during the conversion cycle is the area enclosed by the triangle $A-B-C-A$. The relationship between the input voltage $\Phi_{i n}$ and the maximum 
energy density for a maximum stretch of 5.5 is shown in Figure 2(a). At a particular input voltage, there will be a capacitance value that gives the optimum energy density. Thus, by choosing both the appropriate capacitance and input voltage, the magnitude of the voltage can be controlled so that the DE is still operating within the allowable limits, while also maximizing the energy density. The maximum energy density is calculated to be $1.1 \mathrm{MJ} . \mathrm{m}^{-3}$ (or $1150 \mathrm{~J} \cdot \mathrm{kg}^{-1}$ ) at nominal input voltage, $\Phi_{\text {in }} / H$, of $6.3 \mathrm{kV} \cdot \mathrm{m}^{-1}$, and $C_{\text {ratio }}=C p / C_{D E \text {, max }}=1.3$ $(C p=105 \mathrm{nF}$ for a $0.5 \mathrm{~mm}$ thick, $40 \mathrm{~mm}$ diameter acrylic $\mathrm{DE})$. This value is close to the

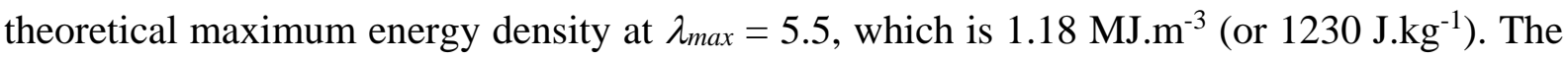
difference between the theoretical maximum energy density and the proposed method is shown as the hatched area above the $A-B-C$ triangle in Figure 1(a). For comparison, the maximum energy density of a constant voltage harvesting scheme, illustrated by the area enclosed by rectangle $d-e-f-g$, is $0.54 \mathrm{MJ} . \mathrm{m}^{-3}$ (or $560 \mathrm{~J}^{\mathrm{kg}} \mathrm{kg}^{-1}$ ). This value is the same as a recent

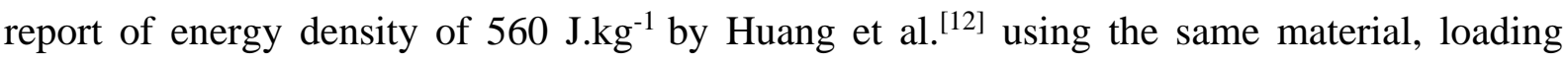
conditions, and harvesting cycle, indicating that further increases in energy density using constant voltage harvesting scheme will only be possible by increasing in the maximum stretch without electrical breakdown or by developing higher breakdown voltage elastomers.

The new harvesting cycle was demonstrated using an equi-biaxial loading configuration and acrylic material, similar to those used in our previous studies. ${ }^{[12]}$ However, the electrical circuit was modified and control of the timing of switches, S1 and S2 was programed. The variation in voltage and current during one electromechanical cycle of the generator operating under the new harvesting scheme is shown in Figure 2(b) for illustration. At the start of the cycle, A', the DE is at a stretch of 2 and has some charge left from the previous cycle at $3 \mathrm{kV}$. Under open circuit conditions, the elastomer is stretched to $\lambda_{\max }=5.5$, corresponding to state A in Figure 1. The capacitance of the DE increases which, at constant charge, decreases the voltage to $50 \mathrm{~V}$. When switch $\mathrm{S} 1$ is then closed, charge flows from 
power supply to the elastomer's electrodes at a constant current of $400 \mu \mathrm{A}$. When the voltage $\Phi_{D E}$ reaches $3 \mathrm{kV}$, corresponding to state $\mathrm{B}$, switch $\mathrm{S} 1$ is opened and simultaneously the servomotor moves back to its starting position, decreases the stretch back to $\lambda_{\min }=2$. The mechanical energy stored in the stretched elastomer is converted to electrical energy as this occurs as charges move from the DE electrodes to the transfer capacitor at a steadily increasing potential. Note the reversal in sign of the current which indicates that the charge moved in the opposite direction in going from B-C compared to the direction during charging in A-B.

An important observation shown in Figure 2(b) is that the voltage increases most rapidly in the initial unloading, roughly in the first third of B-C, but then progressively more slowly. This time dependence is attributed to the viscoelastic behavior of the acrylic material, increasing the response time of the DE. On reaching state C, switch 2 is closed and the remaining charge on the DE flows to the external harvesting circuit. Concurrently, charge also flows (not shown) from the capacitor $C p$ to the external harvesting circuit at the same voltage as that of DE. Once the voltage across the DE and transfer capacitor, $\Phi_{D E}$ and $\Phi_{C p}$, fall to the supply voltage, $3 \mathrm{kV}$, at state $\mathrm{A}^{\prime}$, switch 2 is opened and the harvesting cycle is repeated. Note that if the voltage of DE is allowed to reach zero, then state $\mathrm{A}^{\prime}$ and A will be overlap in voltage-charge plane Figure 3(b). The integrated area under the current, shown shaded in Figure 2(b), is the total charge flow, $Q=\int i d t$. The calculated output charge, $Q_{\text {out }}=217 \mu \mathrm{C}$, is slightly lower than the input charge, $Q_{i n}=227 \mu \mathrm{C}$. This difference is due to charge leakage through the elastomer and loss through the voltage measurement instrument. The latter is calculated at $Q_{\text {disssipated }}=0.9 \mu \mathrm{C}$, which is significantly smaller than the total charge difference of $10 \mu \mathrm{C}$.

The mechanical response for the same electromechanical cycle is shown in Figure 3(a). At the maximum stretch (point A), switch 2 is opened and electrical charge flows from the 
power supply to the DE, decreasing the tension in the elastomer and consequently the load registered by the load cell. It should be noted that the decrease in tension is not only attributed to charges moving, but also to the viscoelastic relaxation of the elastomer. This latter is known from classic load relaxation experiments (not shown) in which the elastomer without any charges is stretched to a constant value and the tension in the elastomer is monitored. The decrease in tension is a loss of restoring force, indicating dissipative losses, and reduces the amount of elastic energy available that can be converted into usable electrical energy. At state $\mathrm{B}$, the mechanical load is decreased, allowing the DE to relax while electrically connected in parallel with the transfer capacitor $C p$. The mechanical cycle is closed when the starting state $\mathrm{A}^{\prime}$ is reached. Again, at this state, the difference in mechanical force between the start and the end of the cycle is a result of the viscoelasticity of the acrylic material. The area enclosed by the curves A'-A-B-C in Figure 3(a) is the mechanical energy input ${ }^{[8,10,12]}$, which in this example had a value of $1.55 \mathrm{~J}$.

The behavior of the generator in the electrical work-conjugate plane, voltage versus charge, is shown in Figure 3(b). The charging characteristic, shown by line A-B, is essentially the same as the one shown in Figure 1(a): a line where the inverse of the slope is the capacitance of the $\mathrm{DE}$ at the maximum stretch. The position of state $\mathrm{C}$ in the work-conjugate plane is the main difference between the ideal harvesting curve shown Figure 1(a) and the experimental curve shown Figure 3(b). In the experiment, state $\mathrm{C}$ is determined by the servomotor position, i.e. at the minimum servomotor position, rather than the minimum stretch of the DE as discussed in the earlier section. Due to viscoelasticity of the acrylic material, the actual stretch of the DE lagged near a stretch of 4 despite the servomotor being fully relaxed - with a significant amount of charge, $90 \mu \mathrm{C}$ or $41 \%$ of the total harvestable charge, was still left on the DE. State C can be seen in the force-stretch work-conjugate plane, Figure 3(a), as an undulation point at stretch around 4, which is consistent with the calculated stretch from the instantaneous capacitance at state $\mathrm{C}$ in Figure 3(b). From the rate of voltage 
increase vs time shown in Figure 2(b), the DE appears in an almost loss of tension condition at state $\mathrm{C}$, a condition to be avoided for the generator mode. However, delaying the activation of switch S2, results in additional loss of charge through the DE thickness and as this occurs at the highest voltages is particularly undesirable. When switch S2 is closed, charge flows from DE (and from the capacitor $C p$ ) to the external harvesting circuits and is marked by the decrease in voltage as the charge of DE decreases. The area between line A-B and the charge axis in Figure 3(b) is the total electrical energy input $(0.38 \mathrm{~J})$ from the power supply whereas the area enclosed by curves A'-A-B-C is the net electrical energy $(0.47 \mathrm{~J})$ generated by the DE. Accounting for the total mass of the DE between the electrodes and the mechanical energy input, the energy density and conversion efficiency of the DE are $780 \mathrm{~J}^{\mathrm{kg}} \mathrm{kg}^{-1}$ and $30 \%$, respectively. The former value is substantially higher than previously reported energy density

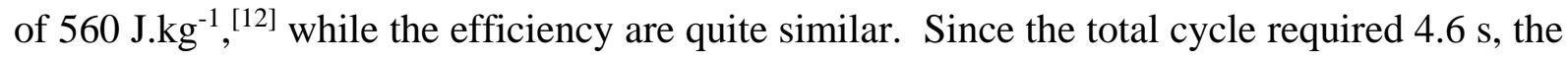
power density is $170 \mathrm{~W} \cdot \mathrm{kg}^{-1}$, which is lower than previously reported value of $280 \mathrm{~J}^{\mathrm{kg}} \mathrm{kg}^{-1}$ from the same reference above. However, the power density of the DE can be easily improved, for example by stopping the discharging process at $3.5 \mathrm{~s}$ without significant penalty to the total harvested energy (see Figure 2(b)) or by decreasing the time required for both stretching and charging.

The key advantage of this new electromechanical cycle is the separation of the mechanical energy storage in the form of elastic energy within the DE from the subsequent mechanical to electrical conversion process. As the storage of mechanical energy is in the form of elastic strain energy, the elastomer can be loaded at any rate - nonuniformly or even intermittently. In contrast, the conversion can occur over a much shorter time, i.e., short charging at high current and sudden relaxation of elastomer, limited only by the mechanical inertia and internal frictional dissipation as well as the electrical time constant. In fact, the shorter the discharge time the better since it minimizes the energy loss associated with charge leakage at high electrical field. In a practical situation harvesting with erratic mechanical 
motions, such as ocean waves, the elastic energy build-up may be captured using a one-way ratcheting mechanism that automatically resets after a preset of movement is reached, corresponding to a prescribed DE stretch.

The transfer capacitor may be replaced with a smart harvesting circuit, which has the ability to control the voltage of the dielectric elastomer based on feedback signals. These feedback signals could include the amount of charge transferred from DE and/or the instantaneous stretch of the DE. The former mechanism operates in a similar manner to the transfer capacitor but with the harvested charges being directly stored into a battery, eliminating the need of the second switch, S2. The latter feedback mechanism could be relatively easy to implement if loss of tension is avoided during operation.

In conclusion, an electromechanical harvesting scheme for maximizing the energy density of dielectric elastomer generators is described. This has been achieved by maximizing the cyclic area in the voltage-charge plane by employing three conditions: (1) charging at a maximum mechanical stretch, (2) the amount of charge placed on the DE is relatively high, and (3) control of the harvesting voltage as a function of charge or stretch using a capacitor connected in parallel with DE. The proposed harvesting scheme has been demonstrated and shows a significant improvement in energy density, making it the highest energy yet reported to date for a dielectric elastomer generator.

\section{Experimental Section}

The acrylic elastomer (VHB 4905, 3M) was coated on both sides using carbon grease (CAT. NO. 846-80G, MG Chemicals) to serve as compliant electrodes. The elastomer thickness was $H=0.5 \mathrm{~mm}$, and the electroded radius, $R_{\mathrm{o}}$, was $2.0 \mathrm{~cm}$ corresponding to a generator mass of $M=0.60 \mathrm{~g}$. Equi-biaxial loading was accomplished by applying radial forces to the circumference of the elastomer sheet through a system of clips, threads and pulleys all loaded by the motion of a linear servomotor (Model SLP35, Nippon Pulse America 
Inc.). The applied force, F, was recorded with a load cell (Model LSB200, Futek, Inc.) attached to the servomotor. Video recording were made from which the radius $R$ of the sheet and the radial stretch, defined as $\lambda=R / R_{\mathrm{o}}$, were obtained. A multichannel voltmeter (model USB-6218, National Instruments), was used to measure the voltages and currents of both the DE and the capacitor $C p$. Note that 1000:1 voltage dividers ( $\mathrm{R}_{\text {total }} 20 \mathrm{G} \Omega$ ) were used to measure the high voltages, while shunt resistors were used to measure the currents (Figure S1). All the data recording as well as the operation of the servomotors and switches were performed using a custom LabVIEW program.

\section{Acknowledgements}

Our research was supported by the National Science Foundation through grant CMMI1333835. SZ is grateful to Fukuoka Institute of Technology for financial support during his sabbatical at Harvard.

[1] R. D. Kornbluh, R. Pelrine, H. Prahlad, A. Wong-Foy, B. McCoy, S. Kim, J. Eckerle, T. Low, MRS Bull. 2012, 37, 246.

[2] R. Pelrine, R. D. Kornbluh, J. Eckerle, P. Jeuck, S. Oh, Q. Pei, S. Stanford, Proc. SPIE 2001, 4329, 148 .

[3] S. Chiba, M. Waki, R. Kornbluh, R. Pelrine, Smart Mater. Struct. 2011, 20, 124006.

[4] S. J. A. Koh, X. Zhao, Z. Suo, Appl. Phys. Lett. 2009, 94, 262902.

[5] C. Graf, J. Maas, D. Schapeler, Proc. SPIE 2010, 7642, 764217.

[6] T. McKay, B. O’Brien, E. Calius, I. Anderson, Smart Mater. Struct. 2010, 19, 55025.

[7] K. Ahnert, M. Abel, M. Kollosche, P. J. Jørgensen, G. Kofod, J. Mater. Chem. 2011, 21,14492 .

[8] S. J. A. Koh, C. Keplinger, T. Li, S. Bauer, Z. Suo, IEEE/ASME Trans. Mechatronics 2011, 16, 33 .

[9] A. N. Gent, Rubber Chem. Technol. 1996, 69, 59.

[10] R. Kaltseis, C. Keplinger, R. Baumgartner, M. Kaltenbrunner, T. Li, P. Machler, R. Schwodiauer, Z. Suo, S. Bauer, Appl. Phys. Lett. 2011, 99, 162903. 
[11] J. Maas, C. Graf, Smart Mater. Struct. 2012, 21, 064006.

[12] J. Huang, S. Shian, Z. Suo, D. R. Clarke, Adv. Funct. Mater. 2013, 23, 5056.

[13] Y. Liu, L. Liu, Z. Zhang, Y. Jiao, S. Sun, J. Leng, Europhys. Lett. 2010, 90, 36004.

[14] C. C. Foo, S. Cai, S. A. Koh, S. Bauer, Z. Suo, J. Appl. Phys. 2012, 111, 034102.

[15] T. Li, S. Qu, W. Yang, J. Appl. Phys. 2012, 112, 034119. 


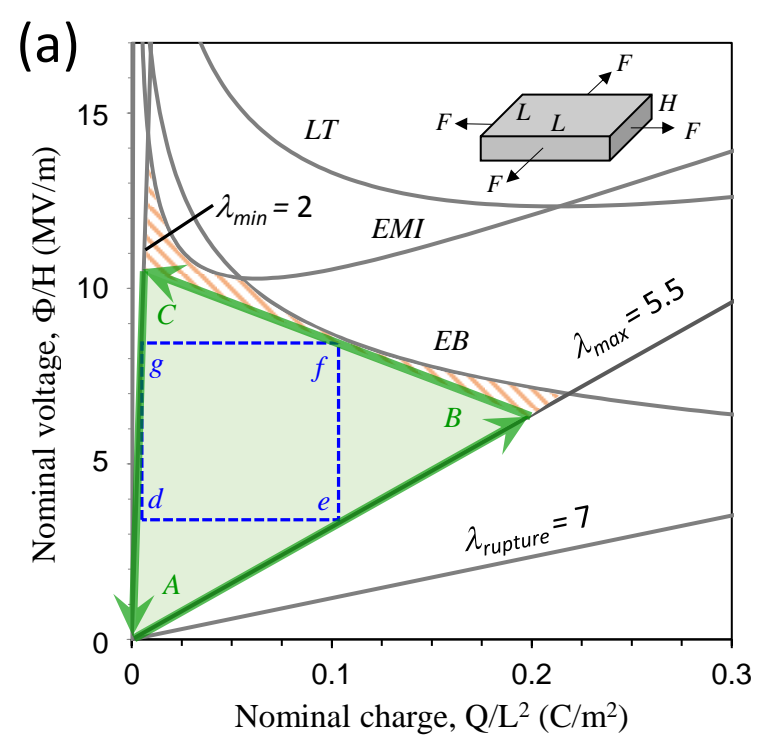

(b)

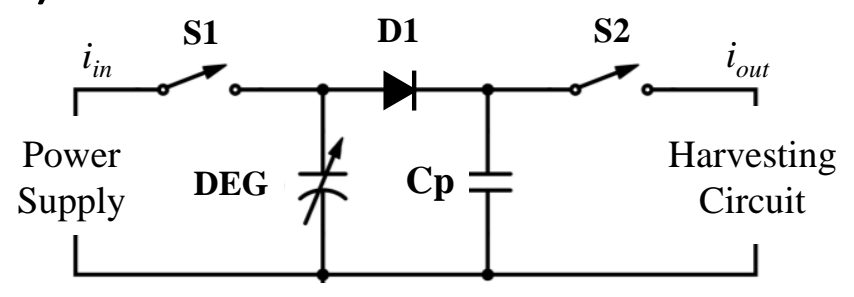

Figure 1. (a) The proposed electromechanical harvesting scheme is shown by the triangle $A$ $B-C$ - $A$ on the voltage-charge work-conjugate plane. Also, shown are the loci of the possible failure modes by electrical breakdown $(E B)$, electromechanical instability $(E M I)$, loss of tension $(L T)$, and rupture stretch $\left(\lambda_{\text {rupture }}\right)$. The diagram is constructed for equibiaxial loading (inset) and for acrylic materials (VHB 4900 series, 3M), and is based on the work by Koh et al. ${ }^{[8]}$ For comparison, the constant-voltage electromechanical harvesting cycle $d-e-f-g$ is also shown. (b) Circuit diagram used to control the electromechanical cycle showing a power supply, the elastomer (DEG), a transfer capacitor (Cp), a diode (D1), a charging switch (S1), harvesting switch (S2), and harvesting circuits block that collect the electrical energy. 

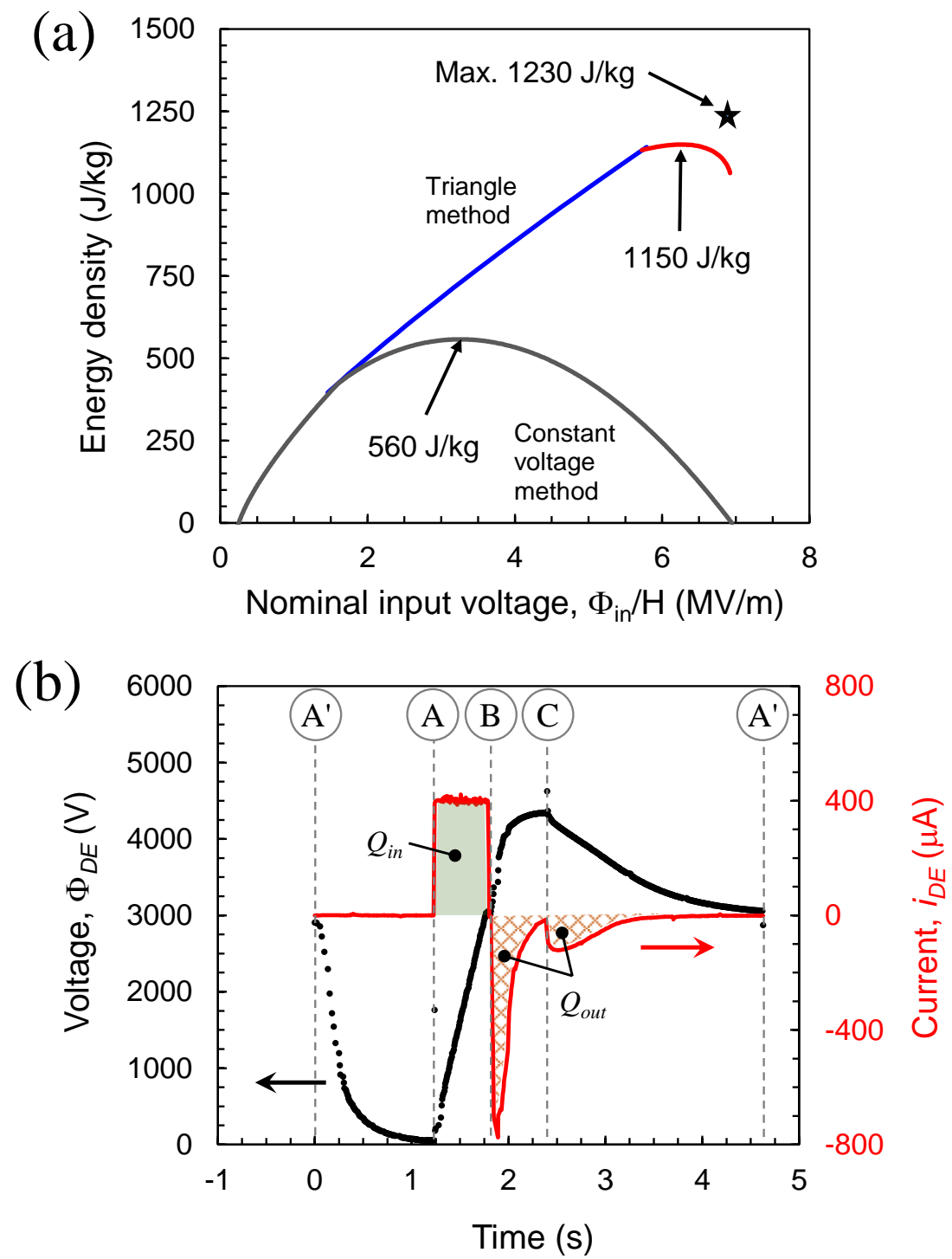

Figure 2. (a) The maximum specific energy density as a function of voltage input ( $\left.\Phi_{\text {in }}\right)$ for both the constant voltage method and the proposed triangle method. Note the blue and red lines indicate the harvesting paths adopted to avoid failure by EMI and EB, respectively. (b) The voltage and current dynamics during one electromechanical harvesting cycle. 

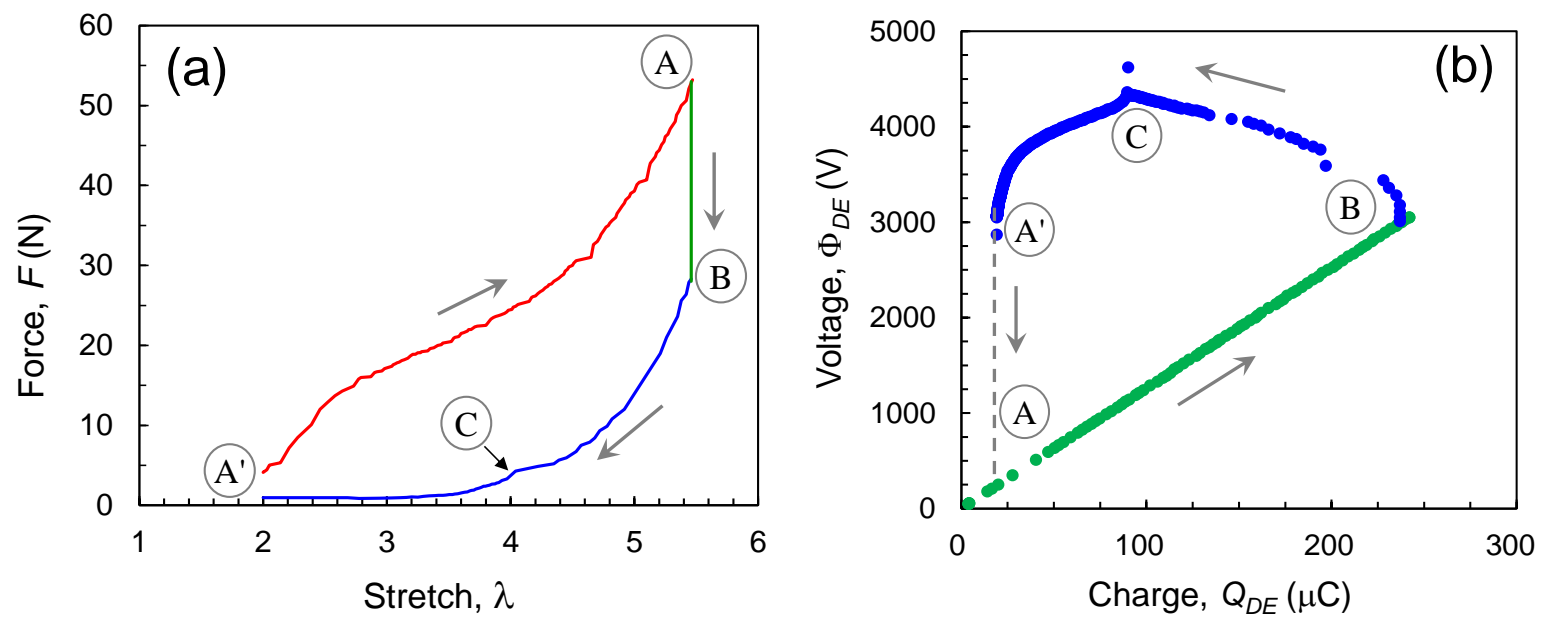

Figure 3. The electromechanical cycle of the dielectric elastomer shown in two workconjugate planes: (a) force-stretch and (b) voltage-charge planes. 


\section{Supporting Information}

\section{Optimizing the Electrical Energy Conversion Cycle of Dielectric Elastomer Generators}

Samuel Shian*, Jiangshui Huang, Shijie Zhu, and David R. Clarke

School of Engineering and Applied Sciences, Harvard University, Cambridge, MA 02138, USA

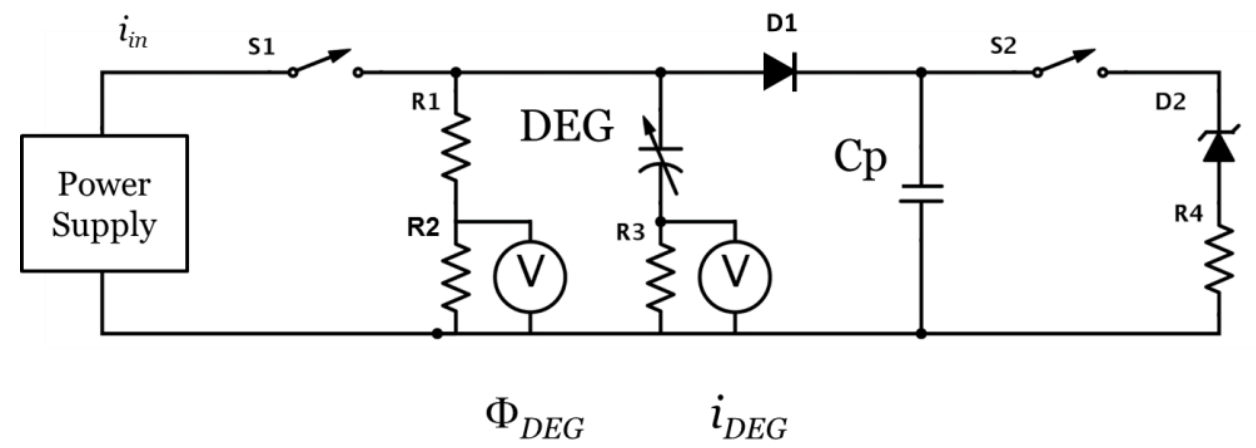

Figure S1. Complete circuit diagram used in the experiments showing a power supply (Trek, model 610E) that supply input charge at constant current; The elastomer (DEG); A transfer capacitor $(\mathrm{Cp})$; The voltage of the DEG is measured using voltage divider R1 (20 G $\Omega$ ) and R2 (20 M $\Omega$ ); The current that flows through DEG is measured through shunt resistor R3 $(10 \mathrm{k} \Omega)$; Diode D1 (NTE517) prevent reverse current flow from Cp to DE; The minimum harvesting voltage is set by the reverse breakdown voltage of zener diodes D2 (1N5271BDO35 x 30; $100 \mathrm{~V} \times 30=3 \mathrm{kV})$ with current limiter R4 $(5 \mathrm{M} \Omega)$.

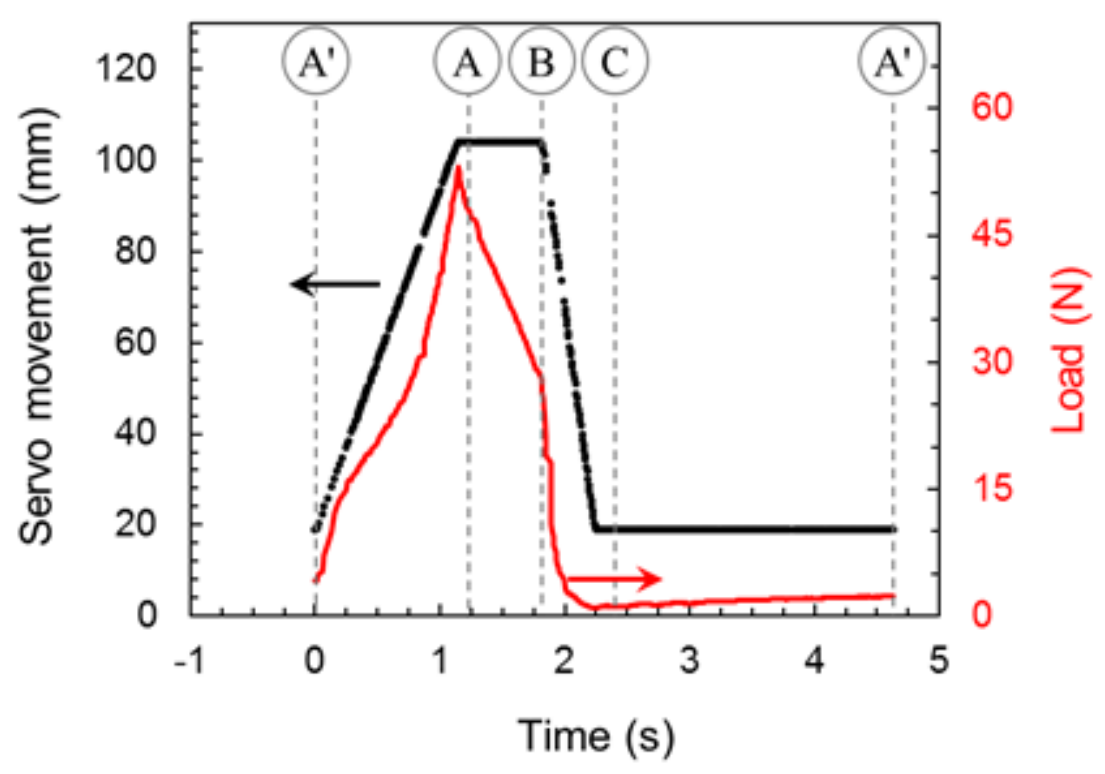

Figure S2. The dynamics of servomotor and load during one electromechanical harvesting cycle. 